Article

\title{
Two Current-Based Methods for the Detection of Bearing and Impeller Faults in Variable Speed Pumps
}

\author{
Vincent Becker ${ }^{1,2}$, Thilo Schwamm ${ }^{1}$, Sven Urschel ${ }^{1, *(1)}$ and Jose Alfonso Antonino-Daviu ${ }^{2}$ (I) \\ 1 University of Applied Sciences Kaiserslautern, Schoenstraße 11, 67659 Kaiserslautern, Germany; \\ vinbec@doctor.upv.es (V.B.); thsc0040@stud.hs-kl.de (T.S.) \\ 2 Technological Institute of Energy, Polytechnic University of Valencia (UPV), Camino de Vera s/n, \\ 46022 Valencia, Spain; joanda@die.upv.es \\ * Correspondence: sven.urschel@hs-kl.de; Tel.: +49-631-3724-2240
}

Citation: Becker, V.; Schwamm, T.;

Urschel, S.; Antonino-Daviu, J.A.

Two Current-Based Methods for the Detection of Bearing and Impeller Faults in Variable Speed Pumps. Energies 2021, 14, 4514. https:// doi.org/10.3390/en14154514

Academic Editor: Ahmed Abu-Siada

Received: 29 June 2021

Accepted: 22 July 2021

Published: 26 July 2021

Publisher's Note: MDPI stays neutral with regard to jurisdictional claims in published maps and institutional affiliations.

Copyright: (c) 2021 by the authors. Licensee MDPI, Basel, Switzerland. This article is an open access article distributed under the terms and conditions of the Creative Commons Attribution (CC BY) license (https:// creativecommons.org/licenses/by/ $4.0 /)$.

\begin{abstract}
The growing number of variable speed drives (VSDs) in industry has an impact on the future development of condition monitoring methods. In research, more and more attention is being paid to condition monitoring based on motor current evaluation. However, there are currently only a few contributions to current-based pump diagnosis. In this paper, two current-based methods for the detection of bearing defects, impeller clogging, and cracked impellers are presented. The first approach, load point-dependent fault indicator analysis (LoPoFIA), is an approach that was derived from motor current signature analysis (MCSA). Compared to MCSA, the novelty of LoPoFIA is that only amplitudes at typical fault frequencies in the current spectrum are considered as a function of the hydraulic load point. The second approach is advanced transient current signature analysis (ATCSA), which represents a time-frequency analysis of a current signal during start-up. According to the literature, ATCSA is mainly used for motor diagnosis. As a test item, a VSD-driven circulation pump was measured in a pump test bench. Compared to MCSA, both LoPoFIA and ATCSA showed improvements in terms of minimizing false alarms. However, LoPoFIA simplifies the separation of bearing defects and impeller defects, as impeller defects especially influence higher flow ranges. Compared to LoPoFIA, ATCSA represents a more efficient method in terms of minimizing measurement effort. In summary, both LoPoFIA and ATCSA provide important insights into the behavior of faulty pumps and can be advantageous compared to MCSA in terms of false alarms and fault separation.
\end{abstract}

Keywords: advanced transient current signature analysis; bearing faults; circulation pump; cracked impeller; impeller clogging; load point-dependent fault indicator analysis; motor current signature analysis

\section{Introduction}

The number of machines operating at variable speed is steadily increasing. According to a market study from 2021, the compound annual growth rate of VSDs from 2020 to 2026 is expected to be 5\% [1]. In addition to the expected increase of VSDs in the automotive, rail vehicle, and renewable energies industries, it is also expected that more and more pumps will be driven at variable speeds. Pumps are among the biggest electrical energy consumers, as they consume $22 \%$ of the electrical motor energy in the European Union [2]. Application examples can be found in cooling, heating, and drinking water systems or in the transport of liquid foodstuffs and chemicals. The advantages of VSDs are high system efficiency, high resource efficiency in production [3], and low currents during start-up of induction motors (IM) [4]. The use of VSD also has an impact on the application of current-based condition monitoring approaches. One issue is that certain frequencies in the current spectrum are influenced by VSD operation, which can lead to false alarms [5]. However, VSD application is also related to some advantages. One advantage is that VSDs equipped with current 
sensors and microcontrollers are a potential platform for implementing automated currentbased condition monitoring algorithms [6,7]. Additionally, VSDs are able to calculate the current hydraulic operation point of a pump [8]. Information about the operation point can be included in diagnosis, which is new compared to the state of the art.

Current-based approaches for motor diagnosis are divided into steady state and transient analysis. The best-known steady state method is MCSA, which is based on spectrum analysis of a current signal of one motor phase [9]. In Park's vector approach (PVA), the patterns of the d-and q-currents are analyzed [10]. Space vector angular fluctuation is a method where the difference between the space vector angle and the angle of an ideal space vector is considered in the frequency domain [11]. A transient analysis that has become widely used in recent years is ATCSA, which is based on time-frequency analysis of the motor current [12,13]. ATCSA has also been applied on VSD-driven motors for the diagnosis of different kinds of faults [4].

There are already a number of papers in the literature focusing on current-based fault detection of pumps. With the help of PVA, a damaged impeller and hydraulic blockage were successfully detected in [14]. The authors of [15] showed that inlet and exit tip faults of an impeller cause a decrease in amplitude at the blade pass frequency (BPF) in MCSA. Additionally, it was shown that a damaged pump impeller causes speed oscillations and can be detected by analyzing the current signal [16]. Clogged impeller channels can lead to a decrease in the current consumption compared to a healthy variant [17] and to an increase in the sideband amplitudes in MCSA [18]. Using an adapted MCSA, a pump operating under the influence of cavitation was detected by analyzing the BPF [19]. In [20], three current-based approaches for the detection of cavitation, dry running, and hydraulic blockage were compared.

The effect by which faults in the motor load influence the motor current can be explained by two approaches: (1) the change in motor behavior in the angular direction of the rotation and (2) the response to air gap modulation caused by displacements and deflections of the motor shaft [21]. The former approach is well-known in applications with load fluctuations. These load torque oscillations are even visible under healthy conditions; for example, in the cement industry [22]. The second approach is mainly explained by eccentricities caused, for example, by bearing damage. Frequency bands influenced by eccentricities are denoted as $f_{\text {ecc }}$. Impeller defects can be explained by the first approach; the influenced frequency bands are denoted as $f_{r, p u m p}$. Despite their different nature, both fault types influence the same frequency bands, which complicates fault separation [23]. Reference [24] shows that impeller clogging mainly influences the same frequencies as a bearing defect. The two frequencies are calculated based on Equation (1) depending on the number of motor pole pairs $p$ and the stator frequency $f_{s}$.

$$
f_{\text {ecc }}=f_{r, p u m p}=\left(1 \pm \frac{1}{p}\right) \cdot f_{s}
$$

Reference [25] shows that multiples of the stator frequency are used to separate clogging and bearing defects from each other. However, the differences in the amplitudes are small. Another option for distinguishing between load oscillations and eccentricities is based on stator current Wigner distributions [23]. However, the disadvantage of this approach is that a fault model must be present. A third approach that enables separating faults is ATCSA. An application example where faults influencing the same frequency bands were separated is shown in [26]. In addition, it has been shown that typical IM faults can be distinguished from load variations $[27,28]$.

In the context of this paper, two current-based approaches are presented. The first approach is LoPoFIA, which represents an adaptation of MCSA. However, in contrast to MCSA, only certain frequencies of the current signal are considered and load points of the pump are included in analysis. The second approach is ATCSA. Both methods are compared to MCSA in terms of fault separation capability and robustness to false alarms. Both methods have the potential for industrial application, especially for application in VSD pumps. 
As a test item, an $800 \mathrm{~W}$ circulation pump was measured in a laboratory test bench, where clogging, a cracked impeller, and bearing defects were analyzed. Circulation pumps are pumps with an integrated VSD, a permanent magnet synchronous motor (PMSM), and a microprocessor unit. Circulation pumps are known to operate only $6 \%$ of their operating time at rated flow and $44 \%$ of their operating time at $25 \%$ of rated flow [29]. For this reason, they represent a good use case for applying ATCSA and LoPoFIA.

The structure of this paper is as follows. In Section 2, we explain the methodology we have used. We show how the two approaches are applied and how they relate to each other. Furthermore, the setup of the experiment, the implemented faults, and the execution of the experiment are presented. The results of LoPoFIA and ATCSA are discussed in Section 3. Section 4 concludes the paper and provides a brief outlook on future work.

\section{Methodology}

\subsection{Implementation of the Methods}

LoPoFIA is a diagnostic method derived from MCSA, so the first steps of both methods are the same. The first step is the normalization of the current signal to the maximum value. This ensures that the maximum amplitude of the frequency domain is $0 \mathrm{~dB}$, which simplifies comparison of the results. After normalization, fast Fourier transformation is applied to obtain the frequency spectrum of the current signal. It is assumed that the captured current signal is stationary and that the valve position remains constant during current measurement. Figure 1a shows the MCSA result of a single load point at a flow of $10 \mathrm{~m}^{3} / \mathrm{h}$ and a head of $6 \mathrm{~m}$. In comparison to MCSA, where the whole spectrum is considered, only two defined frequencies are considered in LoPoFIA, depending on the load point. This is done for the two frequencies calculated based on Equation (1). The two amplitudes at the fault frequencies $f_{r, p u m p-}$ and $f_{r, p u m p}+$ are marked in Figure 1a. For LoPoFIA, these amplitudes are extracted, and each amplitude is displayed depending on the measured flow and head. Consequently, each amplitude has its own plot. In this example, $f_{r, p u m p}+$ is shown at the top of Figure $1 \mathrm{~b}$ and $f_{r, p u m p-}$ at the bottom of Figure 1b. MCSA was applied to each load point in order to extract the load-dependent information of the two fault indicators. The analysis was done with the help of a self-built tool that performs MCSA and calculates the fundamental frequency and the two fault frequencies automatically. To find the maxima at each fault frequency, the tool searches for the maximum value around the calculated frequency within a range of $0.3 \mathrm{~Hz}$. The flow chart of the tool is presented in Figure 2. The number of load points $\mathrm{N}$ equaled 23.

ATCSA was implemented as the second approach. The current signal of the motor performing a start-up was used as a basis for the transformation. An example of the curves of the current signal used is shown in Figure 3a. In order to track the trajectories of the fault frequencies calculated based on Equation (1), short time Fourier transform (STFT) with Gaussian time windowing was applied. The combination of STFT and a Gaussian window is also known as the Gabor transformation, and it was applied based on Equation (2), where $\sigma$ is the deviation parameter, $t_{C}$ and $f_{c}$ are the time and frequency at the center of an atom, and $C_{\sigma}$ is the normalization constant $\left(C_{\sigma}=1 /(\sqrt[4]{\pi} \sqrt{\sigma})\right)$ [4].

$$
\Phi(t)=\left(C_{\sigma} e^{-\frac{\left(t-t_{C}\right)^{2}}{2 \sigma^{2}}}\right) \cdot e^{i 2 \pi f_{c}\left(t-t_{C}\right)}
$$

The ATCSA pattern of an eight-pole motor with eccentricity or load oscillations performing a ramped start-up is shown in Figure $3 b$, with the pronounced sidebands at $(1 \pm 1 / 4)$ times the fundamental frequency. 


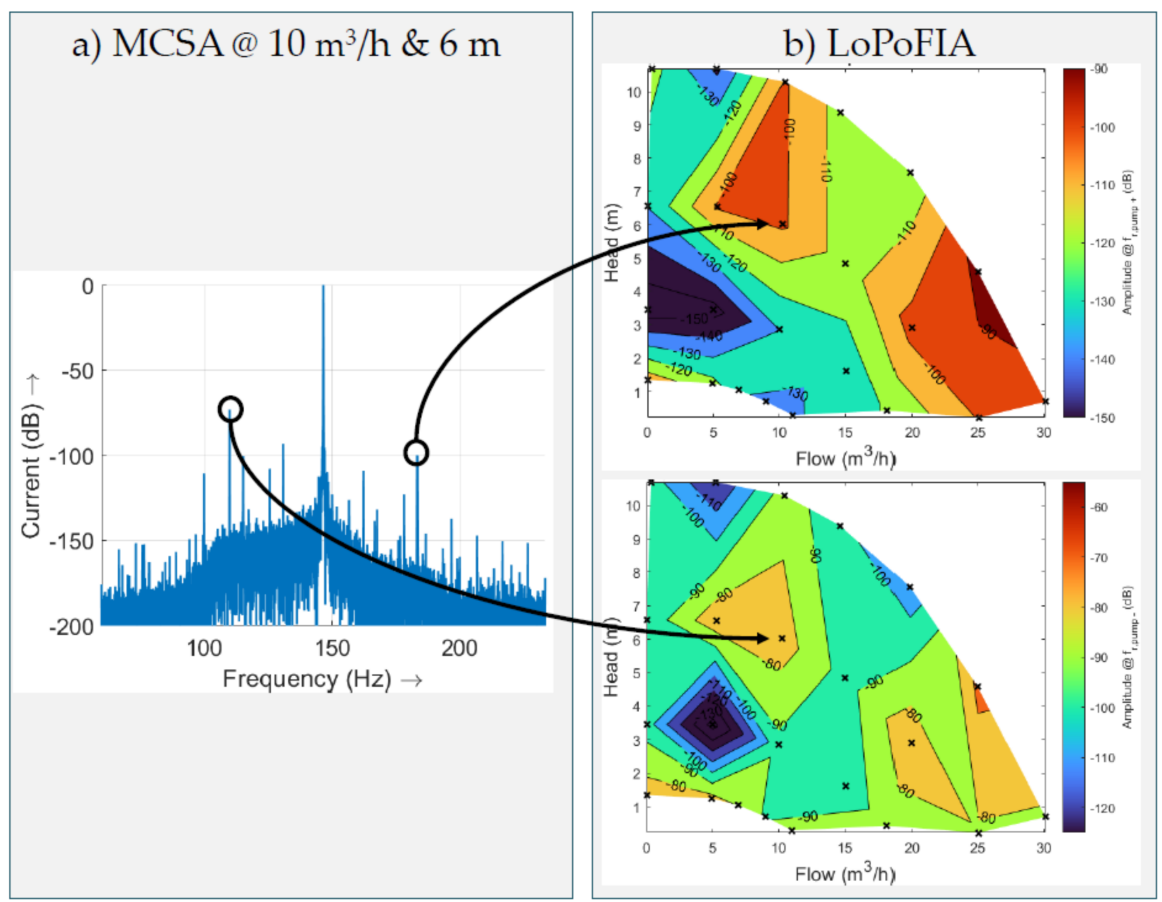

Figure 1. Methodology of feature extraction from (a) MCSA to (b) LoPoFIA.

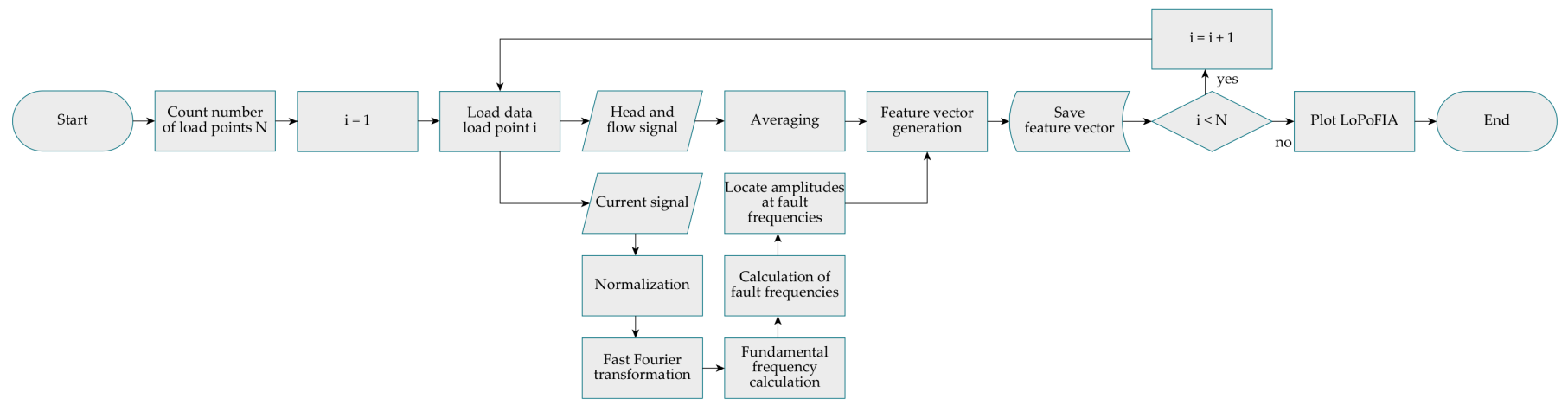

Figure 2. Flow chart of the implementation of the LoPoFIA.
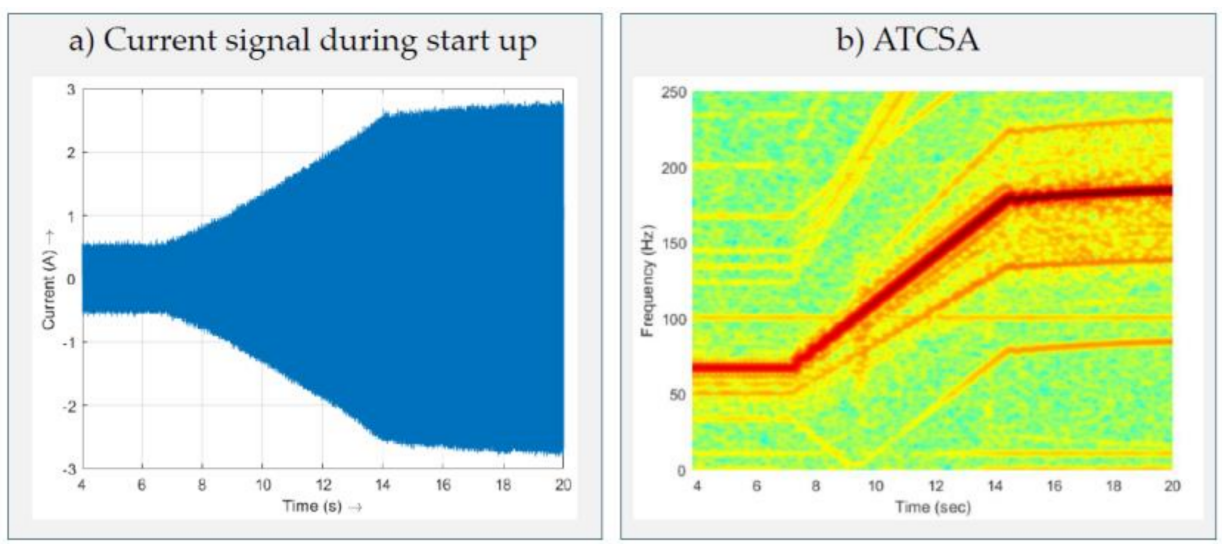

Figure 3. Implementation of the time-frequency transformation; (a) current signal during start-up; (b) ATCSA. 


\subsection{Material}

\subsubsection{The Pump Test Bench}

As our test item, a circulation pump of the type Calio 50-180 with a maximum power of $800 \mathrm{~W}$ was measured in a pump test bench. The pump was designed as a wet-rotor pump, where a tooth coil winding was sealed from the flooded rotor space with the help of a spacer can. The seven-bladed radial impeller was driven by a PMSM with four pole pairs. The rotor shaft was supported by journal bearings. The motor was controlled by an integrated VSD based on field-oriented control, where the speed was set from the test bench computer via a Modbus interface.

The construction of the pump test bench is shown in Figure 4a. It consisted of five vertical strands but only two were used in the context of this measurement. The test item (1), pumping water clockwise, was installed in the middle strand. Shot-off valves (2) were located in the middle strand. With the help of the upper shut-off valve, the flow was set individually depending on the load point. Two IFM PU5415 pressure sensors (3) on the pressure and the suction side measured the pump head. The flow was measured with the help of an Omega FDT-25 ultrasonic flow meter (4) at the right strand. For the acquisition of the analog pressure signals, a NI USB-6363 was installed in the control cabinet (5) on the left of the test bench.
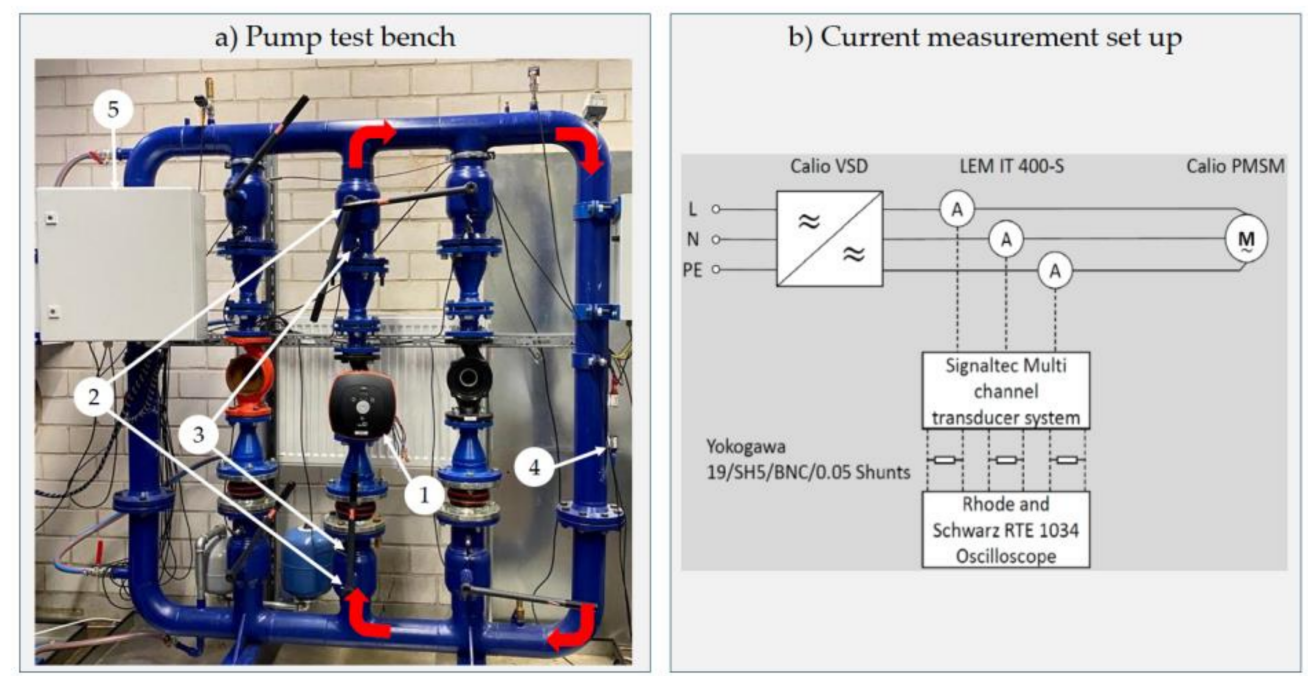

Figure 4. The experimental setup; (a) pump test bench; (b) current measurement setup.

The current measurement was carried out as shown in Figure 4b. LEM IT 400-S current sensors measured the currents of the motor phases that were led to the outside. Via shunts, the currents were captured with the help of a Rohde and Schwarz RTE 1034 oscilloscope. For each measurement, a sampling rate of $10 \mathrm{kHz}$ with a running time of $60 \mathrm{~s}$ was set.

\subsubsection{Investigated Faults}

The two fault types "bearing defect" and "impeller defect" were investigated in this experiment (see Figure 5) The affected components are shown in the scheme in Figure 5a. Additionally, as the healthy variant was measured with the shut-off valve completely closed, these load points represent hydraulic blockage [6,20].

For the manipulation of the bearings, both bearing shells were dismounted and the diameter of the bearing shell was ground down by $0.1 \mathrm{~mm}$ with the help of a lathe. A special pump construction allowed removal of the bearing shells as required. In summary, three combinations of bearing defects were investigated (see Figure $5 b-d$ ). In the first variant, only the A-bearing was changed to a defective bearing, while the B-bearing remained in healthy condition. In the second variant, the bearings were installed the other way round. In the third variant, a defective A-bearing and a defective B-bearing (A- + B-bearing) were installed in the pump. 


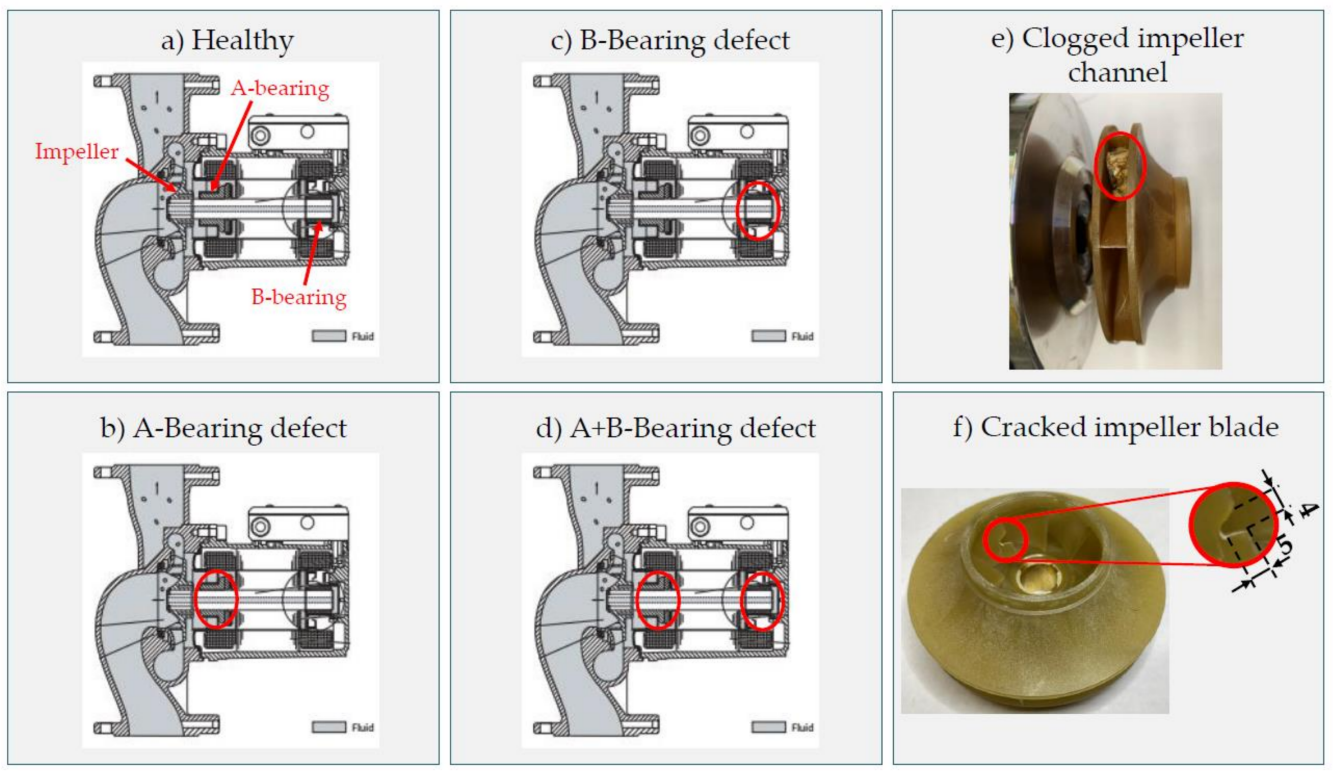

Figure 5. Investigated variants; (a) healthy; (b) A-bearing defect; (c) B-bearing defect; (d) A- + Bbearing defect; (e) clogged impeller channel; (f) cracked impeller blade; (a-d) [30].

Two variants of impeller faults were investigated. In the first variant, one of the seven impeller channels was clogged with polystyrene (see Figure 5e). In order to prove that the impeller channel was clogged during measurement, it was checked afterwards. In the second variant, a part of the impeller blade was cracked out. In Figure $5 f$, the dimensions of the defect are shown.

As every fault variant was measured additionally with the shut-off valves completely closed, these load points were interpreted as a combination of the implemented fault and full hydraulic blockage.

\subsubsection{Experiment Execution}

For the application of the LoPoFIA and ATCSA methods, one measurement campaign for each method was carried out. In each measurement campaign, the six variants depicted in Figure 5 were measured in the test bench presented in Section 2.2.1.

For LoPoFIA, a total of 23 load points were measured by varying the pump speed and flow. While the speed was set via Modbus signal from the test bench computer, the flow was set by adjusting the position of the shut-off valve on the pressure side of the pump. In total, the four speeds 1000, 1600, 2200, and $2800 \mathrm{rpm}$ were measured. Except at the load points at the fully closed shot-off valve, flows lower than $5 \mathrm{~m}^{3} / \mathrm{h}$ were prevented in order to guarantee accuracy of the flow meter $(1 \% @$ flow $>0.2 \mathrm{~m} / \mathrm{s})$.

For ATCSA, start-ups were measured for each of the six variants. As a first step, the flow at a speed of $2800 \mathrm{rpm}$ was set to $20 \mathrm{~m}^{3} / \mathrm{h}$. Afterwards, the speed was set to $1000 \mathrm{rpm}$, which was the initial condition for start-up. While capturing the motor currents, the pump speed was then ramped up from 1000 to $2800 \mathrm{rpm}$.

\section{Results}

In this section, we present and discuss the results of the LoPoFIA and ATCSA of the studied variants.

\subsection{LoPoFIA}

In order to obtain a reference for the faulty variants, the LoPoFIA results of the healthy variant are considered first. Figure 6 depicts the LoPoFIA of the lower sideband (left) and the upper sideband (right). The measured load points are marked with a black $\mathrm{x}$. The four pump curves for the four speeds are visible, starting at $0 \mathrm{~m}^{3} / \mathrm{h}$ at $1.3 \mathrm{~m}, 3.6 \mathrm{~m}, 6.8$ $\mathrm{m}$, and $10.8 \mathrm{~m}$. With increasing flow, the pump curves show typical pump behavior with 
decreasing head. The nominal range of the pump curve is where the product of flow and head has the highest values.
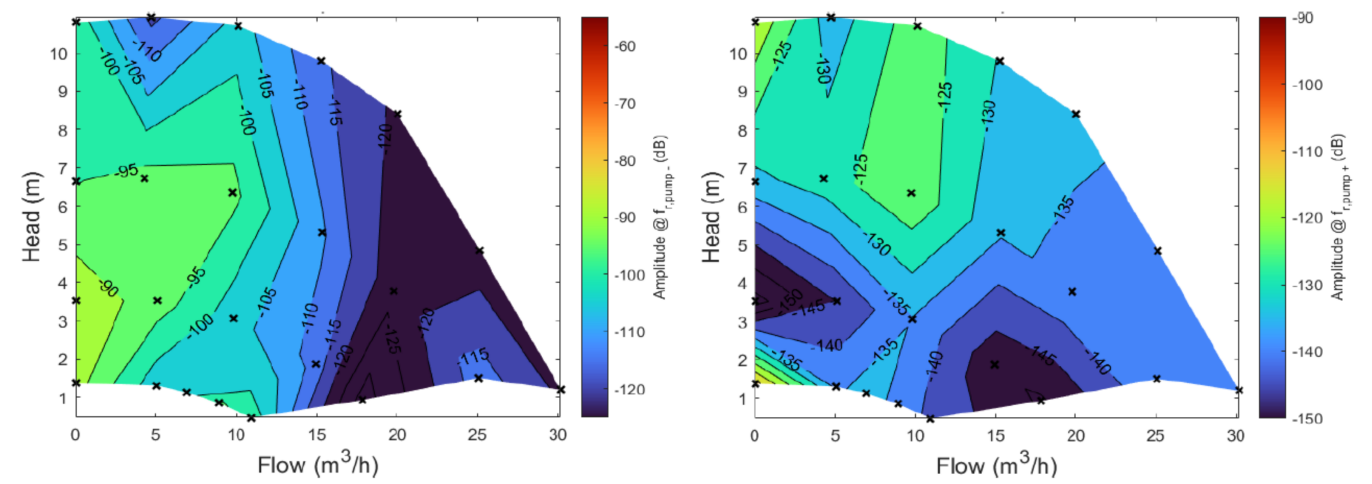

Figure 6. LoPoFIA for the fault indicators $f_{r, p u m p-}$ and $f_{r, p u m p+}$ in healthy condition.

It is remarkable that the lower sideband showed a higher amplitude than the upper sideband. The highest amplitude of the lower sideband was $30 \mathrm{~dB}$ higher than that of the upper sideband. The lower sideband amplitude was mainly dependent on the pump flow. The lowest amplitudes were reached in the high flow range. The upper sideband had a dependence on head and flow. The highest amplitude was located in the medium flow range at higher speeds.

A pipe blockage that allowed no flow in the process was detected with the help of the lower sideband. At zero flow and low speed, the amplitude was the highest. Depending on the application, zero flow can be interpreted differently. When designating the normal operating range, care must be taken not to generate false alarms, as the amplitudes are increased compared to the nominal flow range. In the ranges marked in green, there is a possibility of false negative alarms if decision-making with constant thresholds is used.

To summarize the findings, both flow and head had a certain influence on the amplitude of both sidebands. This is an important fact, especially for decision-making design and for the definition of thresholds.

Next, the influence of bearing defects was investigated. Figures 7 and 8 show the results for a one-sided bearing defect. Figure 9 shows the results for the two-sided bearing defect. If the results of the A-bearing defect are compared with the healthy variant, a similar pattern can be observed. However, the amplitudes were more pronounced for the A-bearing defect. The highest amplitudes of the lower sideband are visible in the low flow range. The maximum $(-75 \mathrm{~dB})$ was reached in the low flow and head range. At the nominal point of the pump, the amplitudes have their minima. Here the difference to the healthy variant becomes small. At the upper sideband, no clear pattern is visible. The highest amplitude was reached at $2800 \mathrm{rpm}$ and $5 \mathrm{~m}^{3} / \mathrm{h}$. At this point, the amplitude increased by $35 \mathrm{~dB}$ compared to the healthy variant.

With the B-bearing defect, both sidebands showed a remarkable pattern. The highest amplitudes were at $5 \mathrm{~m}^{3} / \mathrm{h}$ and at speeds of 2200 and $2800 \mathrm{rpm}$. As for the A-bearing defect, the amplitudes became smaller in the nominal range. A B-bearing defect can be separated from an A-bearing defect in the higher flow range of the lower sideband since an A-bearing defect has higher amplitudes in this flow range.

For the A- + B-bearing defect, the amplitudes of both the lower and upper sidebands increased compared to a one-sided bearing defect and the healthy variant. Also, both sidebands had flow dependence here. At the lower sideband, the highest amplitudes are visible in the lower flow range. The maximum was reached at $0 \mathrm{~m}^{3} / \mathrm{h}$ and $3.5 \mathrm{~m}$. Amplitudes at $0 \mathrm{~m}^{3} / \mathrm{h}$ were interpreted as a combination of a bearing defect and pipe blockage. This combination thus led to the highest amplitudes. As in the case of one-sided bearing defects, the amplitudes decreased at the nominal point. However, the A- + Bbearing defect had an increased amplitude compared to the one-sided bearing defects and the healthy variant at the nominal point. 

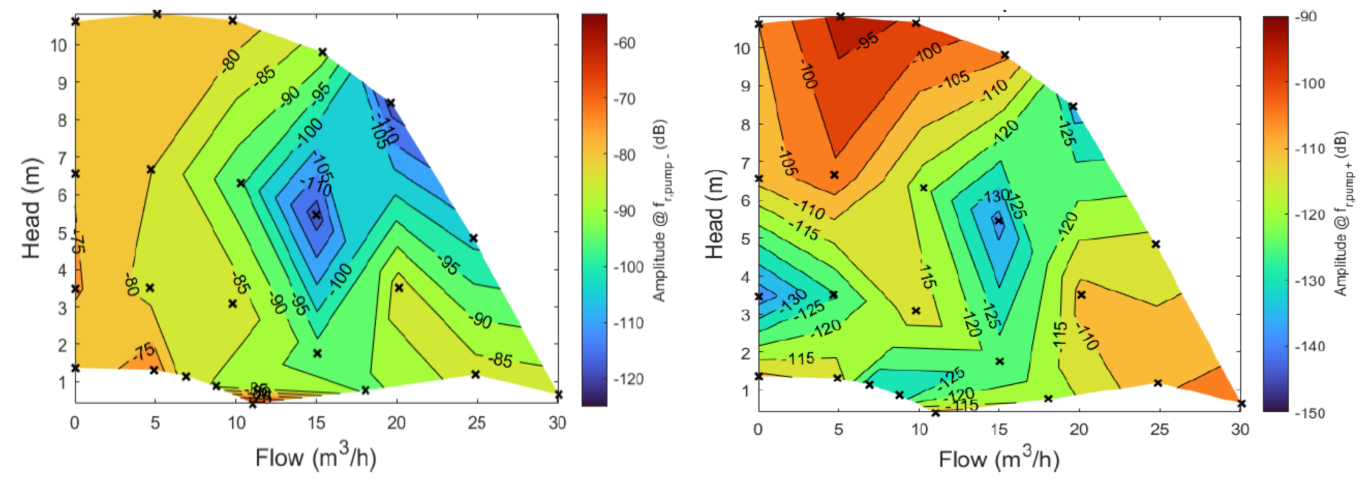

Figure 7. LoPoFIA for the fault indicators $f_{r, p u m p-}$ and $f_{r, p u m p}+$ for a pump with a one-sided bearing defect (A-bearing defect).
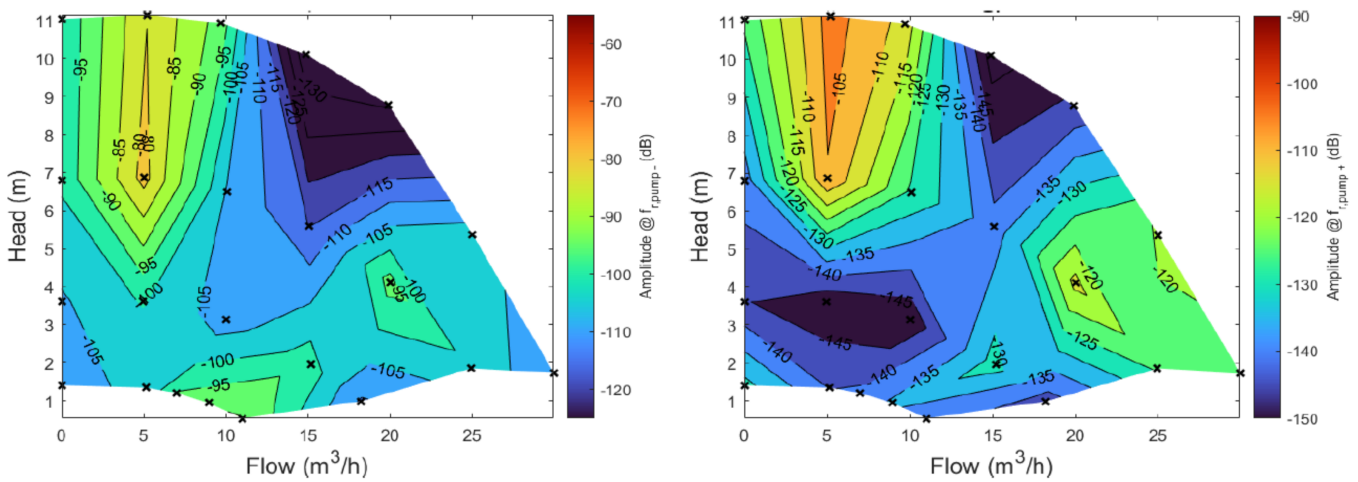

Figure 8. LoPoFIA for the fault indicators $f_{r, p u m p-}$ and $f_{r, p u m p}+$ for a pump with a one-sided bearing defect (B-bearing defect).
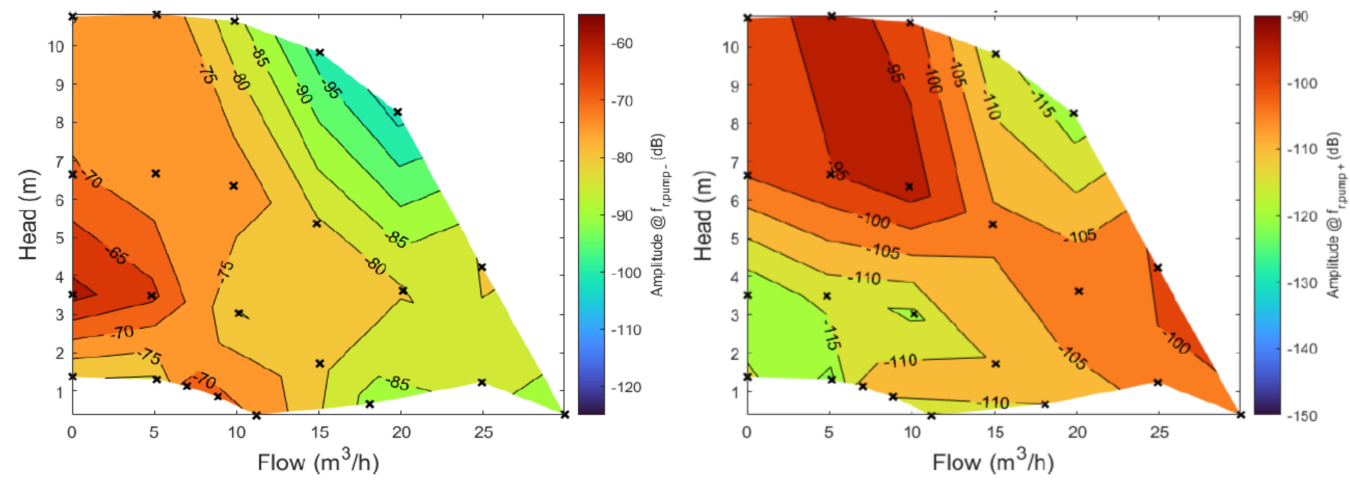

Figure 9. LoPoFIA for the fault indicators $f_{r, p u m p-}$ and $f_{r, p u m p}+$ for a pump with bearing defects on both sides (A- + B-bearing defect).

All bearing defects showed an obvious difference compared to the healthy variant. Furthermore, each bearing defect had its own pattern. An increase of the two amplitudes at $f_{r, \text { pump }}$ and $f_{r, \text { pump }}$ in MCSA was also shown in [24]. The A- + B-bearing defect with the highest fault severity had the highest amplitudes. At the nominal point of the pump, the fault influence on the amplitudes was the smallest. In this area, the risk of false positive alarms increases. If MCSA is applied without including the load points, every load point in the blue areas has a risk of false positive alarms.

Figures 10 and 11 show the results for the impeller faults "clogging" and "cracked blade", respectively. Impeller clogging is considered first. Both sidebands showed a pattern with three pronounced ranges. The first range was at $0 \mathrm{~m}^{3} / \mathrm{h}$ and $1000 \mathrm{rpm}$. This load point, which can be seen as a combination of pipe blockage and clogging, was only slightly increased compared to the healthy variant. The second area was in the medium flow and 
higher speed range. Compared to the healthy variant, in this area the amplitudes increased by $20-25 \mathrm{~dB}$. This result is in accordance with the findings in [18], where an increase of $17 \mathrm{~dB}$, respectively $19 \mathrm{~dB}$, was found at a speed of $2500 \mathrm{rpm}$. However, the A-bearing defect and the A- + B-bearing defect especially also influenced this range. The third range, influenced by clogging, was in the high flow range. Compared to the healthy variant, the amplitudes increased by $40-50 \mathrm{~dB}$. Amplitudes at the lower sideband of $-68 \mathrm{~dB}$ and the upper sideband of $-84 \mathrm{~dB}$ at $25 \mathrm{~m}^{3} / \mathrm{h}$ and $2800 \mathrm{rpm}$ have not been reached by any other fault variant so far. Figure 11 shows that a cracked blade also influenced the two frequencies in LoPoFIA. This confirms the findings in [16], where it was shown that a damaged pump impeller influences the speed and the motor current. The highest amplitudes of both sidebands were reached in the high flow range. At $f_{r, p u m p-}$, a maximum of $-74 \mathrm{~dB}$ was reached, and at $f_{r, p u m p}+$ a maximum of $-96 \mathrm{~dB}$, levels which were not reached by bearing defects in this flow range. The lower sideband had a quite constant amplitude from $-80 \mathrm{~dB}$ to $-90 \mathrm{~dB}$, where the risk of false positive alarms is low. An exception to the risk of false positive alarms is the area marked in blue.
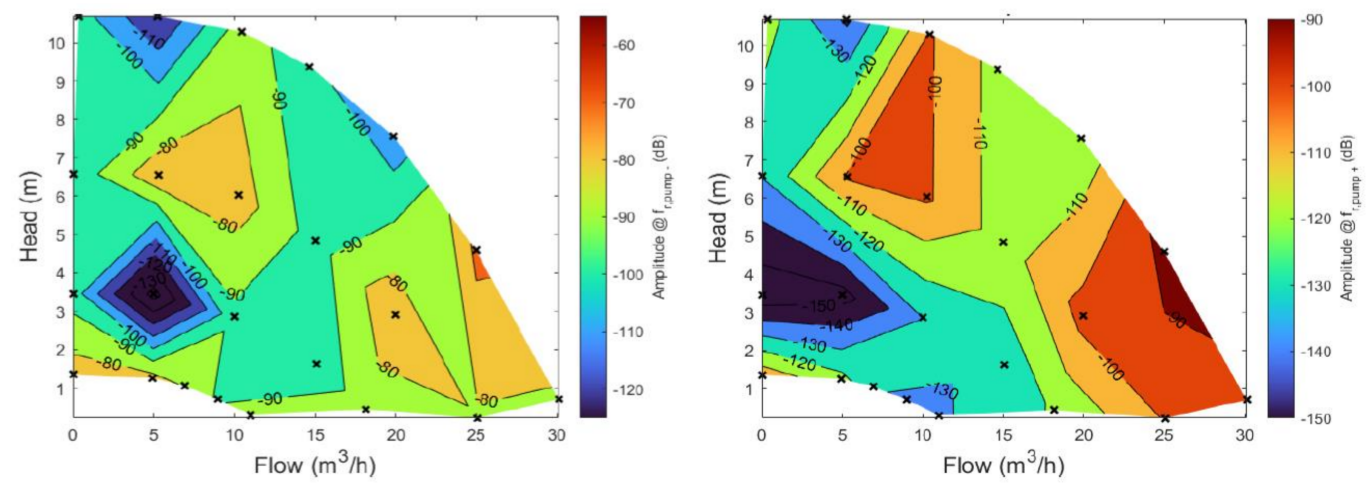

Figure 10. LoPoFIA for the fault indicators $f_{r, \text { pump- }}$ and $f_{r, \text { pump }}$ for a pump with one clogged impeller channel.
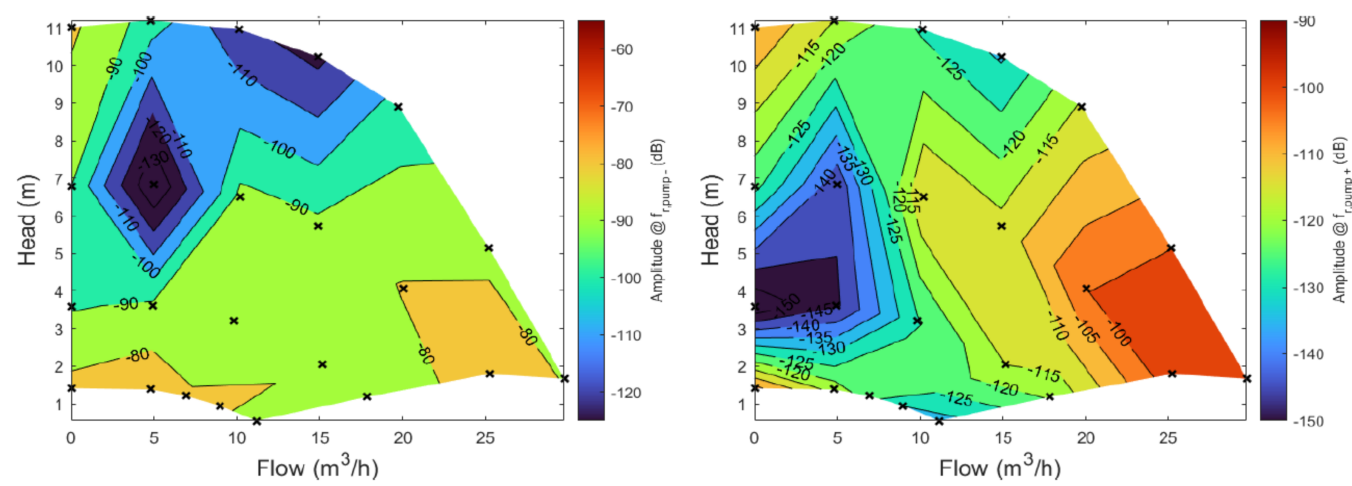

Figure 11. LoPoFIA for the fault indicators $f_{r, \text { pump }}$ and $f_{r, \text { pump }}$ for a pump with a cracked impeller blade.

\subsection{ATCSA}

In this section, the ATCSA results are presented. Figure 12a depicts the results for a start-up transient of the healthy variant from $0-250 \mathrm{~Hz}$. The typical pattern for a ramped start-up is visible starting at the frequency of $66.7 \mathrm{~Hz}(1000 \mathrm{rpm})$ and ending at $186.7 \mathrm{~Hz}$ (2800 rpm). It can also be observed that the current signal is influenced by different kinds of oscillations. One of these oscillations is constantly visible at a frequency of $100 \mathrm{~Hz}$. Considering an MCSA analysis, it is possible that $f_{r, p u m p-}$ or $f_{r, p u m p+}$ equals $100 \mathrm{~Hz}$. In this case, there is a possibility of false negative alarms.

Figure $12 \mathrm{~b}-\mathrm{d}$ show the ATCSA of the different bearing defects analyzed. Compared to the healthy variant, a clear difference is visible. The amplitudes at $f_{e c c}$ and $f_{e c c-}$ show the 
typical trajectories predicted by Equation (1). However, compared to a two-sided bearing defect, the one-sided bearing defect is weaker. On the other hand, for the one-sided bearing defect, an additional amplitude is visible between the eccentricity component and the fundamental component. Another feature for differentiating a one-sided bearing defect from a two-sided bearing defect is that the trajectory of $f_{e c c}+$ starts to be visible later at the one-sided bearing defect.

a) Healthy

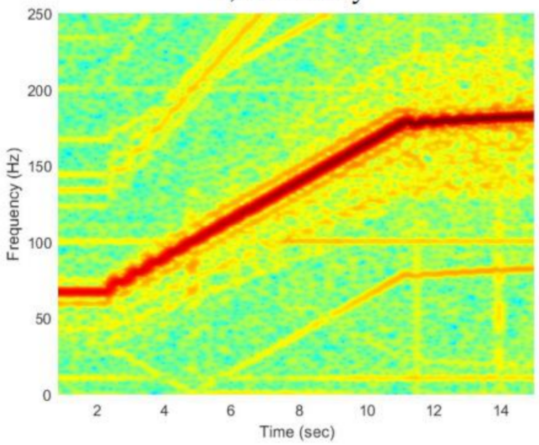

c) B-Bearing defect

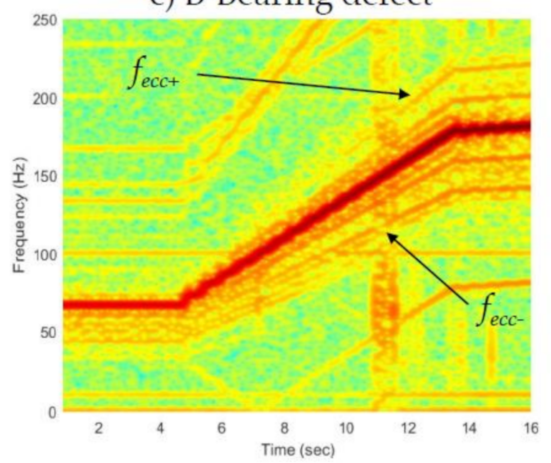

b) A-Bearing defect

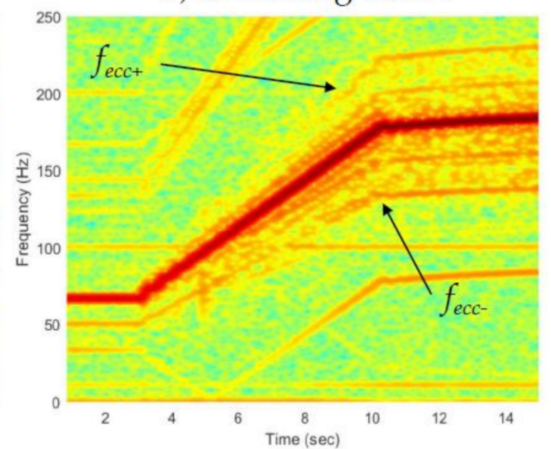

d) A+B-Bearing defect

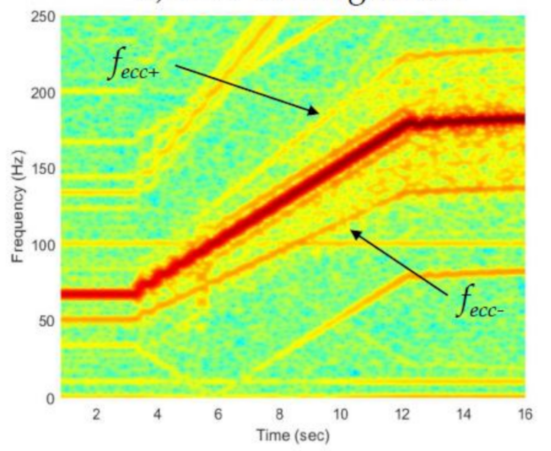

Figure 12. Time-frequency analysis of the stator current in healthy condition and of different variants bearing defects.

Figure 13a depicts the ATCSA of a clogged impeller and Figure 13b that of a cracked blade. Both show the typical pattern for a pump fault, which is clearly different from the healthy variant. As seen in LoPoFIA, both impeller faults produced almost equal amplitudes at the two fault indicators, which is confirmed here again. Compared to a bearing defect, the patterns at $f_{r, p u m p}+$ and $f_{r, p u m p-}$ are slightly more pronounced. $f_{r, p u m p+}$ starts to be visible earlier than in the case of a one-sided bearing fault. However, the difference from a two-sided bearing fault is small.

a) Clogging

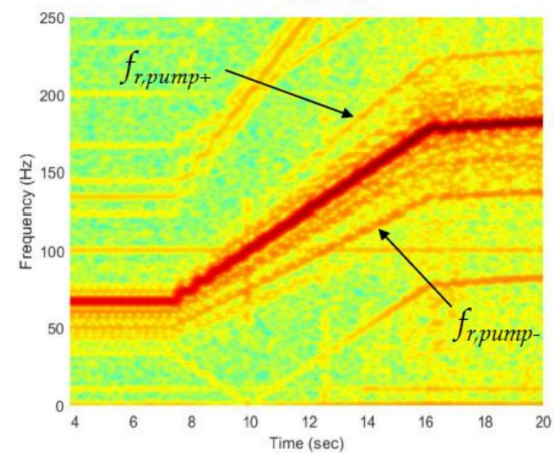

b) Cracked impeller blade

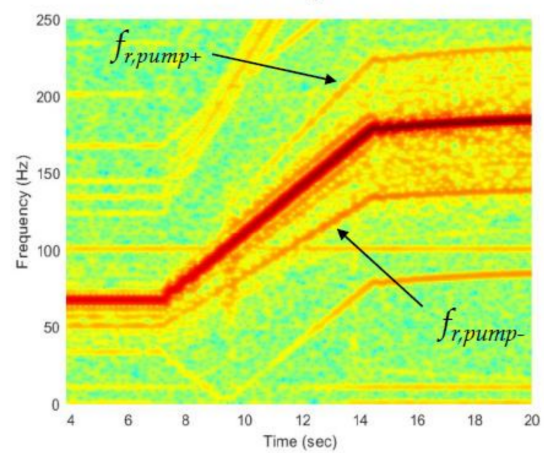

Figure 13. Time-frequency analysis of the stator current with (a) one clogged impeller channel and (b) a cracked impeller blade. 


\section{Conclusions}

Two approaches for current-based pump diagnosis were presented in this paper. The first approach was LoPoFIA, where the amplitudes of typical fault frequencies are considered in relation to load points. LoPoFIA represents an adaptation of the well-known MCSA and is especially suitable for the diagnosis of variable speed pumps. Compared to the previous methods, it is the first approach that relates typical fault indicators to the current operation point. The second approach was ATCSA, which represents a timefrequency analysis of a motor in the transient state.

The LoPoFIA of the healthy variant showed that, at certain load points, the two fault indicators were increased compared to other load points. The amplitude at the lower sideband had an obvious dependence on the flow. When the flow equaled zero, which could be interpreted as the fault "hydraulic blockage", this amplitude was the highest. Consequently, a hydraulic blockage could be detected by analyzing the lower sideband.

The LoPoFIA of every fault variant differed from the healthy variant, and each variant had its own pattern. Clogging and cracked impeller mainly influenced higher flow ranges, while bearing faults mainly influenced lower flow ranges. Consequently, LoPoFIA provides a way to separate impeller and bearing defects. Building on previous approaches, LoPoFIA is a further method for the separation of defects affecting the same frequencies. In addition, we observed that the defects did not affect the fault frequencies at each load point. If MCSA is applied to these load points, this can lead to false positive alarms. This applies, in particular, to defects with a lower degree of severity, such as one-sided bearing defects.

The first step of the practical implementation is the same as for MCSA: the calculation of the amplitudes. Here it must be ensured that the load remains unchanged in the time window of the recording. To counteract this risk, it is recommended to extract the amplitudes of the current signal using methods that are faster than the Fourier transform; for example, reference frame theory [6,7]. The patterns of LoPoFIA can be stored in the microcontroller of a VSD. With respect to the load point, which is estimated or measured, decision-making is improved in comparison to MCSA.

ATCSA showed patterns for all faulty variants that differed from the healthy variant. Consequently, the chance of a false positive alarm can be minimized with the help of ATCSA. However, the differences between impeller and bearing defects are small in ATCSA. Here, LoPoFIA provided better results. Start-ups with different valve positions in the hydraulic system could help in this regard, which will be part of future work. An important finding from ATCSA is that, in circulation pumps, the current signal is under the influence of a load- and speed-independent amplitude at $100 \mathrm{~Hz}$. This fact has to be considered in decision-making designs based on LoPoFIA or MCSA.

In industrial applications, the use of ATCSA depends on whether or not the pump in the system is allowed to perform start-up during operation. For an automated evaluation, the two fault indicators would be recorded during the run-up and the curves compared with the curves of the healthy variant.

In summary, both LoPoFIA and ATCSA are useful methods for the diagnosis of pumps, even for the detection of impeller faults-which is new compared to the current state of the art. Compared to MCSA, LoPoFIA and ATCSA minimize the chance of false alarms. LoPoFIA has its advantages when it comes to the separation of bearing and impeller defects, whereas ATCSA is robust in terms of minimizing false alarms and is an efficient method, since only one measurement is required.

Author Contributions: Conceptualization, J.A.A.-D., S.U., T.S. and V.B.; methodology, software, validation, formal analysis, V.B.; investigation, V.B. and T.S.; resources, S.U.; data curation, V.B.; writing—original draft preparation, V.B.; writing—review and editing, V.B.; visualization, V.B. and T.S.; supervision and project administration, S.U. and J.A.A.-D.; funding acquisition, S.U. All authors have read and agreed to the published version of the manuscript.

Funding: This research was funded by German Federal Ministry for Economic Affairs and Energy, grant number 03ET1613B. 
Institutional Review Board Statement: Not applicable.

Informed Consent Statement: Not applicable.

Conflicts of Interest: The authors declare no conflict of interest.

\begin{tabular}{|c|c|}
\hline \multicolumn{2}{|c|}{ Abbreviations } \\
\hline \multicolumn{2}{|c|}{ The following abbreviations are used in this manuscript: } \\
\hline ATCSA & Advanced transient current signature analysis \\
\hline IM & Induction motor \\
\hline LoPoFIA & Load point-dependent fault indicator analysis \\
\hline MCSA & Motor current signature analysis \\
\hline PMSM & Permanent magnet synchronous motor \\
\hline PVA & Park's vector approach \\
\hline VSD & Variable speed drive \\
\hline
\end{tabular}

\section{References}

1. Businesswire.com. Available online: https://www.businesswire.com/news/home/20210415005587/en/Global-FrequencyConverter-Market-2021-to-2026---Increasing-Demand-for-Water-Wastewater-Treatment-Plants-is-Driving-Growth--ResearchAndMarkets.com (accessed on 30 May 2021).

2. De Almeida, T.A.; Fonsecaa, P.; Falkner, H.; Bertoldi, P. Market transformation of energy-efficient motor technologies in the EU. Energy Policy 2003, 31, 563-575. [CrossRef]

3. Becker, V.; Bold, S.; Urschel, S. Comparison of fixed and variable speed pumps under consideration of manufacturer and operator aspects. In Proceedings of the International Conference EEMODS'19 Energy Efficiency in Motor Driven Systems, Tokyo, Japan, 17-19 September 2019.

4. Fernandez-Cavero, V.; García-Escudero, L.A.; Pons-Llinares, J.; Fernández-Temprano, M.A.; Duque-Perez, O.; Morinigo-Sotelo, D. Diagnosis of Broken Rotor Bars during the Startup of Inverter-Fed Induction Motors Using the Dragon Transform and Functional ANOVA. Appl. Sci. 2021, 11, 3769. [CrossRef]

5. Asad, B.; Vaimann, T.; Kallaste, A.; Belahcen, A.; Rassõlkin, A.; Iqbal, M.N. Broken rotor bar fault detection of the grid and inverter fed induction motor by effective attenuation of the fundamental component. IET Electr. Power Appl. 2019, 13, 2005-2014. [CrossRef]

6. Cruz, S.M.A.; Toliyat, H.A.; Cardoso, A.J.M. DSP implementation of the multiple reference frames theory for the diagnosis of stator faults in a DTC induction motor drive. In Proceedings of the 4th IEEE International Symposium on Diagnostics for Electric Machines, Power Electronics and Drives, Atlanta, GA, USA, 24-26 August 2003; pp. 223-228. [CrossRef]

7. Cruz, S.M.A.; Cardoso, A.J.M. Multiple reference frames theory: A new method for the diagnosis of stator faults in three-phase induction motors. IEEE Trans. Energy Convers. 2005, 20, 611-619. [CrossRef]

8. Leonow, S.; Mönnigmann, M. Soft sensor based dynamic flow rate estimation in low speed radial pumps. In Proceedings of the 2013 European Control Conference (ECC), Zurich, Switzerland, 17-19 July 2013; pp. 778-783. [CrossRef]

9. Thomson, W.T.; Gilmore, R.J. Motor current signature analysis to detect faults in induction motor drive-fundamentals, data interpretation, and industrial case histories. In Proceedings of the Thirty-Second Turbomachinery Symposium; Texas A\&M University. Turbomachinery Laboratories: College Station, TX, USA, 2003.

10. Abitha, M.W.; Rajini, V. Park's vector approach for online fault diagnosis of induction motor. In Proceedings of the 2013 International Conference on Information Communication and Embedded Systems (ICICES), Chennai, India, 21-22 February 2013; pp. 1123-1129. [CrossRef]

11. Kostic-Perovic, D.; Arkan, M.; Unsworth, P. Induction motor fault detection by space vector angular fluctuation. Conference Record of the 2000 IEEE Industry Applications Conference. In Proceedings of the Thirty-Fifth IAS Annual Meeting and World Conference on Industrial Applications of Electrical Energy (Cat. No.00CH37129), Rome, Italy, 8-12 October 2000; pp. 388-394. [CrossRef]

12. Antonino-Daviu, J.; Popaleny, P. Detection of Induction Motor Coupling Unbalanced and Misalignment via Advanced Transient Current Signature Analysis. In Proceedings of the 2018 XIII International Conference on Electrical Machines (ICEM), Alexandroupoli, Greece, 3-6 September 2018; pp. 2359-2364.

13. Antonino-Daviu, J. Electrical Monitoring under Transient Conditions: A New Paradigm in Electric Motors Predictive Maintenance. Appl. Sci. 2020, 10, 6137. [CrossRef]

14. Irfan, M.; Alwadie, A.; Glowacz, A. Design of a novel electric diagnostic technique for fault analysis of centrifugal pumps. Appl. Sci. 2019, 9, 5093. [CrossRef]

15. Tiana, X.; Fenga, G.; Chenb, Z.; Albraika, A.; Guab, F.; Balla, A.D. The investigation of motor current signals from a centrifugal pump for fault diagnosis. In Proceedings of the 27th International Congress of Condition Monitoring and Diagnostic Engineering, Brisbane, Australia, 16-18 September 2014. 
16. Pradhan, P.K.; Roy, S.K.; Mohanty, A.R. Detection of Broken Impeller in Submersible Pump by Estimation of Rotational Frequency from Motor Current Signal. J. Vib. Eng. Technol. 2019, 8, 613-620. [CrossRef]

17. Jahangiri, M.; Roknizadeh, S.A.S. Clogged impeller diagnosis in the centrifugal pump using the vibration and motor current analysis. J. Appl. Comput. Mech. 2018, 4, 310-317. [CrossRef]

18. Becker, V.; Schwamm, T.; Urschel, S.; Antonino-Daviu, J.A. Fault Investigation of Circulation Pumps to Detect Impeller Clogging. Appl. Sci. 2020, 10, 7550. [CrossRef]

19. Stopa, M.M.; Filho, B.J.C.; Martinez, C.B. Incipient detection of cavitation phenomenon in centrifugal Pumps. IEEE Trans. Ind. Appl. 2014, 50, 120-126. [CrossRef]

20. Bold, S.; Urschel, S. Assessment of approaches for technical diagnostic of pump faults with induction motor as transducer. In Proceedings of the 23rd International Conference on Electrical Machines and Systems (ICEMS), Virtual, 24-27 November 2020; pp. 810-815. [CrossRef]

21. Stopa, M.M.; Filho, B.J.C.; Lage, B.L.O. An evaluation of the MCSA method when applied to detect faults in motor driven loads. In Proceedings of the IECON 2010 - 36th Annual Conference on IEEE Industrial Electronics Society, Glendale, CA, USA, 7-10 November 2010; pp. 760-765. [CrossRef]

22. Mabrouk, A.E.; Zouzou, S.E.; Sahraoui, M.; Khelif, S. Discriminating time-varying loads and rotor cage fault in induction motors. In Proceedings of the 2013 9th IEEE International Symposium on Diagnostics for Electric Machines, Power Electronics and Drives (SDEMPED), Valencia, Spain, 27-30 August 2013; pp. 309-316. [CrossRef]

23. Blodt, M.; Regnier, J.; Faucher, J. Distinguishing Load Torque Oscillations and Eccentricity Faults in Induction Motors Using Stator Current Wigner Distributions. IEEE Trans. Ind. Appl. 2009, 45, 1991-2000. [CrossRef]

24. Becker, V.; Schwamm, T.; Urschel, S.; Antonino-Daviu, J.A. Detection of Rotor and Impeller Faults in Wet-rotor Pumps. In Proceedings of the 2020 International Conference on Electrical Machines (ICEM), Virtual, 23-26 August 2020; pp. 1308-1314. [CrossRef]

25. Becker, V.; Schwamm, T.; Urschel, S.; Antonino-Daviu, J.A. Fault Detection of Circulation Pumps on the Basis of Motor Current Evaluation. IEEE Trans. Ind. Appl. 2021. [CrossRef]

26. Park, Y.; Jeong, M.; Lee, S.B.; Daviu, J.A.; Teska, M. Influence of Blade Pass Frequency Vibrations on MCSA-Based Rotor Fault Detection of Induction Motors. IEEE Trans. Ind. Appl. 2017, 53, 2049-2058. [CrossRef]

27. Antonino-Daviu, J.A.; Riera-Guasp, M.; Roger Folch, J.; Molina-Palomares, M.P. Validation of a new method for the diagnosis of rotor bar failures via wavelet transform in industrial induction machines. IEEE Trans. Ind. Appl. 2006, 42, 990-996. [CrossRef]

28. Schoen, R.R.; Habetler, T.G. Evaluation and implementation of a system to eliminate arbitrary load effects in current-based monitoring of induction machines. IEEE Trans. Ind. Appl. 1997, 33, 1571-1577. [CrossRef]

29. Bidstrup, N.; Elburg, M.; Lane, K. Promotion of Energy Efficiency in Circulation Pumps, Final Report. EU SAVE II Project; Environmental Change Institute: Oxford, UK, 2001.

30. Wilo SE "Pump Diagram". Designations were Adjusted by Vincent Becker. Available online: https://creativecommons.org/ licenses/by-sa/3.0/legalcode (accessed on 30 May 2021). 